\title{
A Unified WCET Analysis Framework for Multi-core Platforms
}

\author{
Sudipta Chattopadhyay, Chong Lee Kee, Abhik Roychoudhury \\ National University of Singapore \\ \{sudiptac, cleekee, abhik\}@ comp.nus.edu.sg \\ Timon Kelter, Peter Marwedel \\ Technical University of Dortmund \\ Heiko Falk
Ulm University \\ \{timon.kelter, peter.marwedel\}atu-dortmund. de \\ Heiko.Falk@uni-ulm.de
}

\begin{abstract}
With the advent of multi-core architectures, worst case execution time (WCET) analysis has become an increasingly difficult problem. In this paper, we propose a unified WCET analysis framework for multi-core processors featuring both shared cache and shared bus. Compared to other previous works, our work differs by modeling the interaction of shared cache and shared bus with other basic micro-architectural components (e.g. pipeline and branch predictor). In addition, our framework does not assume a timing anomaly free multicore architecture for computing the WCET. A detailed experiment methodology suggests that we can obtain reasonably tight WCET estimates in a wide range of benchmark programs.
\end{abstract}

\section{INTRODUCTION}

Hard real time systems require absolute guarantees on program execution time. Worst case execution time (WCET) has therefore become an important problem to address. WCET of a program depends on the underlying hardware platform. Therefore, to obtain a safe upper bound on WCET, the underlying hardware need to be modeled. However, performance enhancing micro-architectural features of a processor (e.g. cache, pipeline) make WCET analysis a very challenging task.

With the rapid growth of multi-core architectures, it is quite evident that the multi-core processors are soon going to be adopted for real time system design. Although multicore processors are aimed for improving performance, they introduce additional challenges in WCET analysis. Multicore processors employ shared resources. Two meaningful examples of such shared resources are shared cache and shared bus. The presence of a shared cache requires the modeling of inter-core cache conflicts. On the other hand, the presence of a shared bus introduces variable bus access latency to accesses to shared cache and shared main memory. The delay introduced by shared cache conflict misses and shared bus accesses is propagated by different pipeline stages and affects the overall execution time of a program. WCET analysis is further complicated by a commonly known phenomenon called timing anomalies [1]. In the presence of timing anomalies, a local worst case scenario may not lead to the WCET of the overall program. As an example, a cache hit rather than a cache miss may lead to the
WCET of the entire program. Therefore, we cannot always assume a cache miss or maximum bus delay as the worst case scenario, as the assumptions are not just imprecise, but they may also lead to an unsound WCET estimation. A few solutions have been proposed which model the shared cache and/or the shared bus ([2], [3], [4], [5], [6]) in isolation, but all of these previous solutions ignore the interactions of shared resources with important micro-architectural features such as pipelines and branch predictors.

In this paper, we propose a WCET analysis framework for multi-core platforms featuring both a shared cache and a shared bus. In contrast to previous work, our analysis can efficiently model the interaction of the shared cache and bus with different other micro-architectural features (e.g. pipeline, branch prediction). A few such meaningful interactions include the effect of shared cache conflict misses and shared bus delays on the pipeline, the effect of speculative execution on the shared cache etc. Moreover, our analysis framework does not rely on a timing-anomaly free architecture and gives a sound WCET estimate even in the presence of timing anomalies. In summary, the central contribution of this paper is to propose a unified analysis framework that features most of the basic micro-architectural components (pipeline, (shared) cache, branch prediction and shared bus) in a multi-core processor.

Our analysis framework deals with timing anomalies by representing the timing of each pipeline stage as an interval. The interval covers all possible latencies of the corresponding pipeline stage. The latency of a pipeline stage may depend on cache miss penalties and shared bus delays. On the other hand, cache and shared bus analysis interact with the pipeline stages to compute the possible latencies of a pipeline stage. Our analysis is context sensitive - it takes care of different procedure call contexts and different microarchitectural contexts (i.e. cache and bus) when computing the WCET of a single basic block. Finally, WCET of the entire program is formulated as an integer linear program (ILP). The formulated ILP can be solved by any commercial solver (e.g. CPLEX) to get the whole program's WCET.

We have implemented our framework in an extended version of Chronos [7], a freely available, open-source, single- 
core WCET analysis tool. To evaluate our approach, we have also extended a cycle accurate simulator [8] with both shared cache and shared bus support. Our experiments with moderate to large size benchmarks from [9] show that we can obtain tight WCET estimates for most of the benchmarks in a wide range of micro-architectural configurations.

\section{RELATED WORK}

Research in single-core WCET analysis has started a few decades ago. Initial works used only integer linear programming (ILP) for both micro-architectural modeling and path analysis [10]. However, the work proposed in [10] faces scalability problems due to the explosion in number of generated ILP constraints. In [11], a novel approach has been proposed, which employs abstract interpretation for micro-architectural modeling and ILP for path analysis. Subsequently, an iterative fixed-point analysis has been proposed in [12] for modeling advanced micro-architectural features such as out-of-order and superscalar pipelines. A different paper by the same set of authors [13] has proposed an ILP-based modeling of branch predictors. Our baseline framework is built upon the technique proposed in [12], [13].

Although there has been a significant progress in singlecore WCET analysis research, little has been done so far in WCET analysis for multi-cores. Multi-core processors employ shared resources (e.g. shared cache, shared bus), which gives rise to a new problem for modeling intercore conflicts. A few solutions have already been proposed for analyzing a shared cache [2], [3], [14]. All of these approaches extend the abstract interpretation based cache analysis proposed in [11]. However, in contrast to our proposed framework, these approaches model the shared cache in isolation, assume a timing-anomaly-free architecture and ignore the interaction with different other micro-architectural features (e.g. pipeline and branch prediction). On the other hand, separated shared bus analysis has been proposed in [15], [5], [4]. None of these works model the interactions with pipeline and branch prediction. Additionally, [15] and [4] both assume a timing-anomaly-free architecture.

A recent approach [6] has combined abstract interpretation and model checking for analyzing private cache and shared bus, respectively. However, it is unclear whether such a combination would remain scalable in the presence of a shared cache and other micro-architectural features.

To eliminate the problem of pessimism in multi-core WCET analysis, researchers have proposed predictable multi-core architectures [16] and predictable execution models by code transformations [17]. However, we argue that these approaches are orthogonal to the idea of this paper and our idea in this paper can be used to pinpoint the source of overestimation in multi-core WCET analysis.

In summary, there has been little progress on multi-core WCET analysis by modeling individual micro-architectural components (e.g. shared cache, shared bus). Our work differs

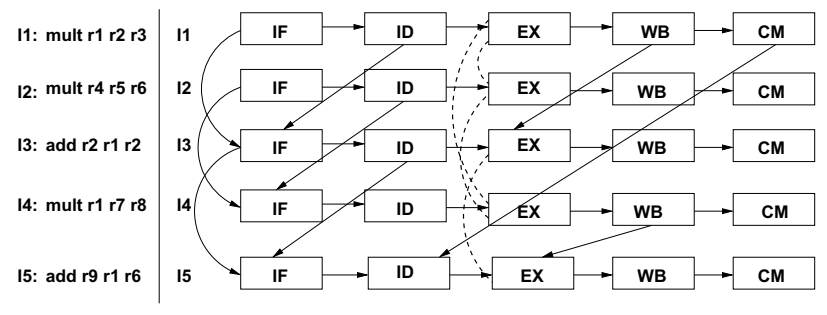

Figure 1. Execution graph for the example program in a 2-way superscalar processor with 2-entry instruction fetch queue and 4-entry reorder buffer. Solid edges show the dependency between pipeline stages, whereas the dotted edges show the contention relation.

from all previous works by proposing a unified framework, which is able to analyze the most basic micro-architectural components and their interactions in a multi-core processor.

\section{BACKGROUND}

Pipeline modeling through execution graphs: The central idea of pipeline modeling revolves around the concept of the execution graph [12]. The execution graph is constructed for each basic block in the program control flow graph (CFG). For each instruction in the basic block, the corresponding execution graph contains a node for each of the pipeline stages. We assume a five stage pipeline instruction fetch (IF), decode (ID), execution (EX), write back (WB) and commit (CM). Edges in the execution graph capture the dependencies among pipeline stages; either due to resource constraints (instruction fetch queue size, reorder buffer size etc.) or due to data dependency (read after write hazard). The timing of each node in the execution graph is represented by an interval, which covers all possible latencies suffered by the corresponding pipeline stage.

Figure 1 shows a snippet of assembly code and the corresponding execution graph. The example assumes a 2way superscalar processor with 2-entry instruction fetch queue (IFQ) and 4-entry reorder buffer (ROB). Since the processor is a 2-way superscalar, instruction I3 cannot be fetched before the fetch of I1 finishes. This explains the edge between IF nodes of I1 and I3. On the other hand, since IFQ size is 2, IF stage of I3 cannot start before ID stage of I1 finishes (edge between ID stage of I1 and IF stage of I3). Note that I3 is data dependent on I1 and similarly, I5 is data dependent on I4. Therefore, we have edges from WB stage of I1 to EX stage of I3 and also from WB stage of I4 to EX stage of I5. Finally, as ROB size is 4, I1 must be removed from ROB (i.e. committed) before I5 can be decoded. This explains the edge from CM stage of I1 to ID stage of I5.

A dotted edge in the execution graph (e.g. the edge between EX stage of I2 and I4) represents contention relation (i.e. a pair of instructions which may contend for the same functional unit). Since I2 and I4 may contend for the same functional unit (multiplier), they might delay each other due to contention. The pipeline analysis is iterative. Analysis starts without any timing information and assumes 


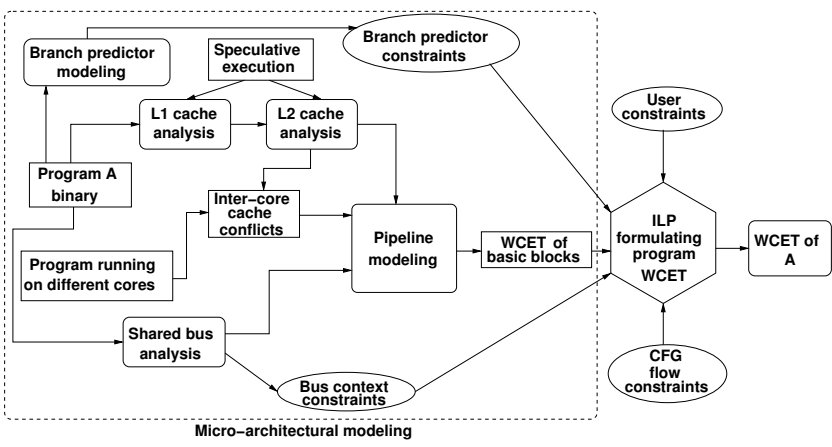

Figure 2. Overview of our analysis framework

that all pairs of instructions which use same functional units and can coexist in the pipeline, may contend with each other. In the example, therefore, the analysis starts with $\{(\mathrm{I} 1, \mathrm{I} 2),(\mathrm{I} 2, \mathrm{I} 4),(\mathrm{I} 1, \mathrm{I} 4),(\mathrm{I} 3, \mathrm{I} 5)\}$ in the contention relation. After one iteration, the timing information of each pipeline stage is obtained and the analysis may rule out some pairs from the contention relation if their timing intervals do not overlap. With this updated contention relation, the analysis is repeated and subsequently, a refined timing information is obtained for each pipeline stage. Analysis is terminated when no further elements can be removed from the contention relation. WCET of the code snippet is then given by the worst case completion time of the CM node for I5.

\section{OVERVIEW OF OUR ANALYSIS}

Figure 2 gives an overview of our analysis framework. Each processor core is analyzed at a time by taking care of the inter-core conflicts generated by all other cores. Figure 2 shows the analysis flow for some program $A$ running on a dedicated processor core. The overall analysis can broadly be classified into two separate phases: 1) micro-architectural modeling and 2) path analysis. In micro-architectural modeling, the timing behavior of different hardware components is analyzed (as shown by the big dotted box in Figure 2). We use abstract interpretation (AI) based cache analysis [11] to categorize memory references as all-hit $(\mathrm{AH})$ or all-miss (AM) in L1 and L2 cache. A memory reference is categorized $\mathrm{AH}(\mathrm{AM})$ if the resulting access is always a cache hit (miss). If a memory reference cannot be categorized as $\mathrm{AH}$ or AM, it is categorized as unclassified (NC). In the presence of a shared L2 cache, categorization of a memory reference may change from $\mathrm{AH}$ to $\mathrm{NC}$ due to the inter-core conflicts [3]. Moreover, as shown in Figure 2, L1 and L2 cache analysis has to consider the effect of speculative execution when a branch instruction is mispredicted (refer to Section VII for details). Similarly, the timing effects generated by the mispredicted instructions are also taken into account during the iterative pipeline modeling (refer to [12] for details). The shared bus analysis computes the bus context under which an instruction can execute. The outcome of cache analysis and shared bus analysis is used to compute the latency of different pipeline stages during the analysis of the pipeline (refer to Section V for details). Pipeline modeling computes the WCET of each basic block. WCET of the entire program is formulated as maximizing the objective function of a single integer linear program (ILP). WCETs of individual basic blocks are used to construct the objective function of the formulated ILP. The constraints of the ILP are generated from the structure of the program control flow graph (CFG), micro-architectural modeling (branch predictor and shared bus) and additional user given constraints (e.g. loop bound). The modeling of the branch predictor generates constraints to bound the execution count of mispredicted branches (for details refer to [13]). On the other hand, constraints generated for bus contexts bound the execution count of a basic block under different bus contexts (for details, refer to Section VI). Path analysis finds the longest feasible program path from the formulated ILP through implicit path enumeration (IPET). Any ILP solver (e.g. CPLEX) can be used for IPET and for deriving the whole program's WCET.

System and application model: We assume a multicore processor with each core having a private L1 cache. Additionally, multiple cores share an L2 cache. The extension of our framework for more than two levels of caches is straightforward. If a memory block is not found in L1 or L2 cache, it has to be fetched from the main memory. Any memory transaction to L2 cache or main memory has to go through a shared bus. For shared bus, we assume a TDMA based round robin arbitration policy, where a fixed length bus slot is assigned to each core. We also assume fully separated caches and buses for instruction and data memory. Therefore, the data references do not interfere with the instruction references. In this work, we only model the effect of instruction caches. However, the data cache effects can be considered in a similar fashion. Since we consider only instruction caches, the cache miss penalty (computed from cache analysis) directly affects the instruction fetch (IF) stage of the pipeline. We do not consider self modifying code and therefore, we do not need to model the coherence traffic. Finally, we consider the $L R U$ cache replacement policy and non-inclusive caches only.

\section{INTERACTION OF SHARED RESOURCES WITH PIPELINE}

Let us assume each node $i$ in the execution graph is annotated with the following timing parameters, which are computed iteratively:

- earliest $\left[t_{i}^{\text {ready }}\right]$ : Earliest ready time of node $i$.

- earliest $\left[t_{i}^{\text {start }}\right]$ : Earliest start time of node $i$.

- earliest $\left[t_{i}^{f \text { inish }}\right]$ : Earliest finish time of node $i$.

- latest $\left[t_{i}^{\text {ready }}\right]$ : Latest ready time of node $i$.

- latest $\left[t_{i}^{\text {start }}\right]$ : Latest start time of node $i$.

- latest $\left[t_{i}^{f i n i s h}\right]$ : Latest finish time of node $i$.

Therefore, the active time span of node $i$ can be represented by the interval $\left[\right.$ earliest $\left[t_{i}^{\text {ready }}\right]$, latest $\left.\left[t_{i}^{\text {finish }}\right]\right]$. In the following sections, we shall discuss how the presence of a 
shared cache and a shared bus affects the timing information of different pipeline stages.

\section{A. Interaction of shared cache with pipeline}

Let us assume $C H M C_{i}^{L 1}\left(C H M C_{i}^{L 2}\right)$ denotes the AH/AM/NC cache hit-miss classification of an IF node $i$ in L1 (shared L2) cache. Further assume that $E_{i}$ denotes the possible latencies of an $I F$ node $i$ without considering any shared bus delay. $E_{i}$ can be defined as follows:

$$
E_{i}=\left\{\begin{array}{l}
1, \quad \text { if } C H M C_{i}^{L 1}=A H ; \\
L A T^{L 1}+1, \\
\text { if } C H M C_{i}^{L 1}=A M \wedge C H M C_{i}^{L 2}=A H ; \\
L A T^{L 1}+L A T^{L 2}+1, \\
\text { if } C H M C_{i}^{L 1}=A M \wedge C H M C_{i}^{L 2}=A M ; \\
{\left[L A T^{L 1}+1, L A T^{L 1}+L A T^{L 2}+1\right],} \\
\text { if } C H M C_{i}^{L 1}=A M \wedge C H M C_{i}^{L 2}=N C ; \\
{\left[1, L A T^{L 1}+1\right],} \\
\text { if } C H M C_{i}^{L 1}=N C \wedge C H M C_{i}^{L 2}=A H ; \\
{\left[1, L A T^{L 1}+L A T^{L 2}+1\right], \quad \text { otherwise. }}
\end{array}\right.
$$

where $L A T^{L 1}$ and $L A T^{L 2}$ represent the fixed L1 and L2 cache miss latencies respectively. Note that the intervalbased representation captures the possibilities of both a cache hit and a cache miss in case of an NC categorized cache access. Therefore, the computation of $E_{i}$ can also deal with the architectures that exhibit timing anomalies.

\section{B. Interaction of shared bus with pipeline}

Let us assume that we have a total of $\mathcal{C}$ cores and the TDMA based round robin scheme assigns a slot length $S_{l}$ to each core. Therefore, the length of one complete round is $S_{l} \mathcal{C}$. We begin with the following definitions which are used throughout the paper:

Definition 5.1: (TDMA offset) A TDMA offset at a particular time $T$ is defined as the relative distance of $T$ from the last scheduled round. Therefore, at time $T$, the TDMA offset can be precisely defined as $T \bmod S_{l} \mathcal{C}$.

Definition 5.2: (Bus context) A Bus context for a particular execution graph node $i$ is defined as the set of TDMA offsets reaching/leaving the corresponding node. For each execution graph node $i$, we track the incoming bus context (denoted $O_{i}^{i n}$ ) and the outgoing bus context (denoted $O_{i}^{\text {out }}$ ).

For a task executing in core $p$ (where $0 \leq p<\mathcal{C}$ ), latest $\left[t_{i}^{\text {finish }}\right]$ and earliest $\left[t_{i}^{\text {finish }}\right]$ are computed for an $I F$ execution graph node $i$ as follows:

$$
\begin{aligned}
\text { latest }\left[t_{i}^{\text {finish }}\right] & =\text { latest }\left[t_{i}^{\text {start }}\right]+\text { max }_{-} l a t_{p}\left(O_{i}^{i n}, E_{i}\right) \\
\text { earliest }\left[t_{i}^{\text {finish }}\right] & =\text { earliest }\left[t_{i}^{\text {start }}\right]+\text { min_lat }_{p}\left(O_{i}^{\text {in }}, E_{i}\right)
\end{aligned}
$$

Note that max_lat , min_lat $_{p}$ are not constants and depend on the incoming bus context $\left(O_{i}^{i n}\right)$ and the set of possible latencies of $I F$ node $i\left(E_{i}\right)$ in the absence of a shared bus. max_lat $_{p}$ and min_lat $_{p}$ are defined as follows:

$$
\max \_l a t_{p}\left(O_{i}^{i n}, E_{i}\right)=\left\{\begin{array}{l}
1, \quad \text { if } C H M C_{i}^{L 1}=A H ; \\
\max _{o \in O_{i}^{i n}, t \in E_{i}} \Delta_{p}(o, t), \text { otherwise. }
\end{array}\right.
$$

$$
\text { min_lat }_{p}\left(O_{i}^{i n}, E_{i}\right)=\left\{\begin{array}{l}
1, \quad \text { if } C H M C_{i}^{L 1} \neq A M ; \\
\min _{o \in O_{i}^{i n}, t \in E_{i}} \Delta_{p}(o, t), \text { otherwise. }
\end{array}\right.
$$

In the above, $E_{i}$ represents the set of possible latencies of an $I F$ node $i$ in the absence of shared bus delay (refer to Equation 1). Given a TDMA offset $o$ and latency $t$ in the absence of shared bus delay, $\Delta_{p}(o, t)$ computes the total delay (including shared bus delay) faced by the IF stage of the pipeline. $\Delta_{p}(o, t)$ can be defined as follows (similar to [4] or [5]):

$$
\Delta_{p}(o, t)=\left\{\begin{array}{l}
t, \text { if } p S_{l} \leq o+t \leq(p+1) S_{l} \\
t+p S_{l}-o, \text { if } o<p S_{l} \\
t+(\mathcal{C}+p) S_{l}-o, \text { otherwise. }
\end{array}\right.
$$

In the following, we shall now show the computation of incoming and outgoing bus contexts (i.e. $O_{i}^{\text {in }}$ and $O_{i}^{\text {out }}$ respectively) for an execution graph node $i$.

Computation of $O_{i}^{\text {out }}$ from $O_{i}^{i n}$ : The computation of $O_{i}^{\text {out }}$ depends on $O_{i}^{i n}$, on the possible latencies of execution graph node $i$ (including shared bus delay) and on the contention suffered by the corresponding pipeline stage. In the modeled pipeline, inorder stages (i.e. IF, ID, WB and CM) do not suffer from contention. But the out-of-order stage (i.e. EX stage) may experience contention when it is ready to execute (i.e. operands are available) but cannot start execution due to the unavailability of a functional unit. Worst case contention period of an execution graph node $i$ can be denoted by the term latest $\left[t_{i}^{\text {start }}\right]-$ latest $\left[t_{i}^{\text {ready }}\right]$. For best case computation, we conservatively assume the absence of contention. Therefore, for a particular core $p(0 \leq p<\mathcal{C})$, we compute $O_{i}^{\text {out }}$ from the value of $O_{i}^{i n}$ as follows:

$O_{i}^{\text {out }}=\left\{\begin{array}{l}u\left(O_{i}^{i n}, E_{i}+\left[0, \text { latest }\left[t_{i}^{\text {start }}\right]-\text { latest }\left[t_{i}^{\text {ready }}\right]\right]\right), \quad \text { if } i=E X ; \\ u\left(O_{i}^{i n}, \bigcup_{o \in O_{i}^{i n}, t \in E_{i}} \Delta_{p}(o, t)\right), \quad \text { if } i=I F ; \\ u\left(O_{i}^{\text {in }}, E_{i}\right), \text { otherwise. }\end{array}\right.$

Here, $u$ denotes the update function on TDMA offset set with a set of possible latencies of node $i$ and is defined as follows:

$$
u(O, X)=\bigcup_{o \in O, t \in X}\left\{(o+t) \bmod S_{l} \mathcal{C}\right\}
$$

Note that $E_{i}+\left[0\right.$, latest $\left[t_{i}^{\text {start }}\right]-$ latest $\left.\left[t_{i}^{\text {ready }}\right]\right]$ captures all possible latencies suffered by the execution graph node $i$, taking care of contentions as well. Therefore, $O_{i}^{\text {out }}$ captures all possible TDMA offsets exiting node $i$, when the same node is entered with bus context $O_{i}^{i n}$. More precisely, assuming that $O_{i}^{i n}$ represents an over-approximation of the incoming bus context at node $i$, the computation by Equation 7 ensures that $O_{i}^{\text {out }}$ represents an over-approximation of the outgoing bus context from node $i$.

Computation of $O_{i}^{i n}$ : The value of $O_{i}^{i n}$ depends on the value of $O_{j}^{\text {out }}$, where $j$ is a predecessor of node $i$ in the execution graph. If pred $(i)$ denotes all the predecessors of node $i$, clearly, $\cup_{j \in \operatorname{pred}(i)} O_{j}^{\text {out }}$ gives a sound approximation of $O_{i}^{i n}$. However, it is important to observe that not all predecessors in the execution graph can propagate TDMA offsets to node $i$. Recall that the edges in the execution graph represent dependency (either due to resource constraints or due to true data dependences). Therefore, node $i$ in the execution graph can only start when all the nodes in 
$\operatorname{pred}(i)$ have finished. Consequently, the TDMA offsets are propagated to node $i$ only from the predecessor $j$, which finishes immediately before $i$ is ready. Nevertheless, our static analyzer may not be able to compute a single predecessor that propagates TDMA offsets to node $i$. However, for two arbitrary execution graph nodes $j 1$ and $j 2$, if we can guarantee that earliest $\left[t_{j 2}^{\text {finish }}\right]>$ latest $\left[t_{j 1}^{\text {finish }}\right]$, we can also guarantee that $j 2$ finishes later than $j 1$. By capturing this property, we can compute $O_{i}^{i n}$ as follows:

$$
O_{i}^{\text {in }}=\bigcup\left\{O_{j}^{\text {out }} \mid j \in \operatorname{pred}(i) \wedge \operatorname{earliest}\left[t_{\text {pmax }}^{\text {finish }}\right] \leq \operatorname{latest}\left[t_{j}^{\text {finish }}\right]\right\}
$$

where pmax is a predecessor of $i$ such that latest $\left[t_{\text {pmax }}^{\text {finish }}\right]=\max _{j \in \operatorname{pred}(i)}$ latest $\left[t_{j}^{\text {finish }}\right]$. Therefore, $O_{i}^{i n}$ captures all possible outgoing TDMA offsets from the predecessor nodes that are possibly finished latest. Given that the value of $O_{j}^{\text {out }}$ is an over-approximation of the outgoing bus context for each predecessor $j$ of $i$, Equation 9 gives an over-approximation of the incoming bus context at node $i$. Finally, Equation 7 and Equation 9 together ensure a sound computation of the bus contexts at the entry and exit of each execution graph node.

\section{WCET COMPUTATION OF A BASIC BLOCK}

\section{A. Execution context of a basic block}

Computing bus context without loops: In the previous section, we have discussed the pipeline modeling of a basic block $B$ in isolation. However, to correctly compute the execution time of $B$, we need to consider 1) contentions (for functional units) and data dependencies among instructions prior to $B$ and instructions in $B ; 2$ ) contentions among instructions after $B$ and instructions in $B$. Set of instructions before (after) $B$ which directly affect the execution time of $B$ is called the prologue (epilogue) of $B$ [12]. $B$ may have multiple prologues and epilogues due to the presence of multiple program paths. However, the size of any prologue or epilogue is bounded by the total size of IFQ and ROB. To distinguish the execution contexts of a basic block $B$, execution graphs are constructed for each possible combination of prologues and epilogues of $B$. Each execution graph of $B$ contains the instructions from $B$ itself (called body) and the instructions from one possible prologue and epilogue. Assume we compute the incoming (outgoing) bus context $O_{i}^{\text {in }}(p, e)\left(O_{i}^{\text {out }}(p, e)\right)$ at body node $i$ for prologue $p$ and epilogue $e$ (using the technique described in Section V). After we finish the analysis of $B$ for all possible combinations of prologues and epilogues, we compute an over-approximation of $O_{i}^{\text {in }}\left(O_{i}^{\text {out }}\right)$ by merge operation: $O_{i}^{\text {in }}=\bigcup_{p, e} O_{i}^{\text {in }}(p, e)$ and $O_{i}^{\text {out }}=\bigcup_{p, e} O_{i}^{\text {out }}(p, e)$. Clearly, $O_{i}^{\text {in }}\left(O_{i}^{\text {out }}\right)$ captures an over-approximation of the bus context at the entry (exit) of node $i$, irrespective of any prologue or epilogue of $B$.

Computing bus context in the presence of loops: In the presence of loops, a basic block can be executed with different bus contexts at different iterations of the loop. The bus contexts at different iterations depend on the set of instructions which can propagate TDMA offsets across loop

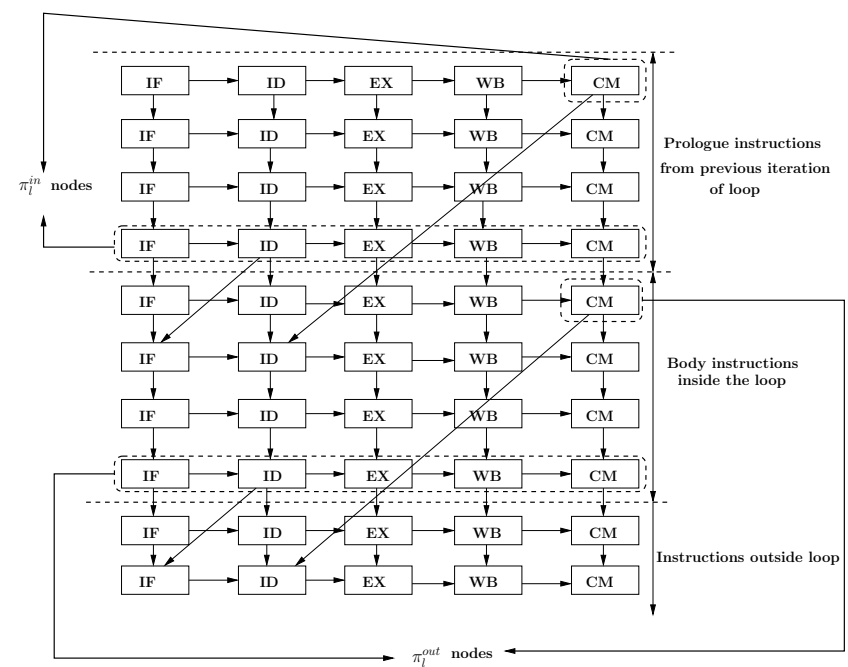

Figure 3. $\pi_{l}^{\text {in }}$ and $\pi_{l}^{\text {out }}$ nodes shown with the example of a sample execution graph. $\pi_{l}^{i n}$ nodes propagate bus contexts across iterations, whereas, $\pi_{l}^{\text {out }}$ nodes propagate bus contexts outside of loop.

iterations. For each loop $l$, we compute two sets of nodes $-\pi_{l}^{i n}$ and $\pi_{l}^{\text {out }} . \pi_{l}^{i n}$ are the set of pipeline stages which can propagate TDMA offsets across iterations, whereas, $\pi_{l}^{\text {out }}$ are the set of pipeline stages which could propagate TDMA offsets outside of the loop. Therefore, $\pi_{l}^{i n}$ corresponds to the pipeline stages of instructions inside $l$ which resolve loop carried dependency (due to resource constraints, pipeline structural constraints or true data dependency). On the other hand, $\pi_{l}^{\text {out }}$ corresponds to the pipeline stages of instructions inside $l$ which resolve the dependency of instructions outside of $l$. Figure 3 demonstrates the $\pi_{l}^{\text {out }}$ and $\pi_{l}^{\text {in }}$ nodes for a sample execution graph. The bus context at the entry of all nonfirst loop iterations can be captured as $\left(O_{x 1}^{i n}, O_{x 2}^{i n}, \ldots, O_{x n}^{i n}\right)$ where $\pi_{l}^{i n}=\{x 1, x 2, \ldots, x n\}$. The bus context at the first iteration is computed from the bus contexts of instructions prior to $l$ (using the technique described in Section V). Finally, $O_{x i}^{\text {out }}$ for any $x i \in \pi_{l}^{\text {out }}$ can be responsible for affecting the execution time of any basic block outside of $l$.

\section{B. WCET computation under multiple bus contexts}

Foundation: As discussed in the preceding, a basic block inside some loop may execute under different bus contexts. In this section, we shall show how the execution count of different bus contexts can be bounded by generating additional ILP constraints. These additional ILP constraints are eventually fed to the global ILP formulation. We begin with the following notations to discuss our technique:

$\Omega_{l}$ : The set of all bus contexts that may reach loop $l$ in any iteration.

$\Omega_{l}^{s}$ : The set of all bus contexts that may reach loop $l$ at first iteration. Clearly, $\Omega_{l}^{s} \subseteq \Omega_{l}$. Moreover, if $l$ is contained inside some outer loop, $l$ would be invoked more than once. As a result, $\Omega_{l}^{s}$ may contain more than one element. 
$G_{l}^{s}$ : For each $s_{0} \in \Omega_{l}^{s}$, we build a flow graph $G_{l}^{s}=\left(V_{l}^{s}, F_{l}^{s}\right)$ where $V_{l}^{s} \subseteq \Omega_{l}$. The graph $G_{l}^{s}$ captures the transitions among different bus contexts across loop iterations. An edge $f_{w_{1} \rightarrow w_{2}}=\left(w_{1}, w_{2}\right) \in F_{l}^{s}$ exists (where $w_{1}, w_{2} \in \Omega_{l}$ ) if and only if $l$ can be entered with bus context $w_{1}$ at some iteration $n$ and with bus context $w_{2}$ at iteration $n+1$. Note that $G_{l}^{s}$ cannot be infinite, as we have only finitely few bus contexts that are the nodes of $G_{l}^{s}$.

$M_{l}^{w}$ : Number of times the body of loop $l$ is entered with bus context $w \in \Omega_{l}$ in any iteration.

$M_{l}^{w_{1} \rightarrow w_{2}}$ : Number of times $l$ can be entered with bus context $w_{1}$ at some iteration $n$ and with bus context $w_{2}$ at iteration $n+1$ (where $w_{1}, w_{2} \in \Omega_{l}$ ). Clearly, if $f_{w_{1} \rightarrow w_{2}} \notin$ $F_{l}^{s}$ for any flow graph $G_{l}^{s}, M_{l}^{w_{1} \rightarrow w_{2}}=0$.

Construction of $G_{l}^{s}$ : For each loop $l$ and for each $s_{0} \in \Omega_{l}^{s}$, we construct a flow graph $G_{l}^{s}$. Initially, $G_{l}^{s}$ contains a single node representing bus context $s_{0} \in \Omega_{l}^{s}$. After analyzing all the basic blocks inside $l$ (using the technique described in Section V), we may get a new bus context at some node $i \in \pi_{l}^{i n}$ (recall that $\pi_{l}^{i n}$ are the set of execution graph nodes that may propagate bus context across loop iterations). As a byproduct of this process, we also get the WCET of all basic blocks inside $l$ when the body of $l$ is entered with bus context $s_{0}$. Let us assume that for any $s \in \Omega_{l} \backslash \Omega_{l}^{s}$ and $i \in \pi_{l}^{i n}, s(i)$ represents the bus context $O_{i}^{i n}$. Suppose we get a new bus context $s_{1} \in \Omega_{l}$ after analyzing the body of $l$ once. Therefore, we add an edge from $s_{0}$ to $s_{1}$ in $G_{l}^{s}$. We continue expanding $G_{l}^{s}$ until $s_{n}(i) \subseteq s_{k}(i)$ for all $i \in \pi_{l}^{i n}$ and for some $1 \leq k \leq n-1$ (where $s_{n} \in \Omega_{l}$ represents the bus context at the entry of $l$ after it is analyzed $n$ times). In this case, we finish the construction of $G_{l}^{s}$ by adding a backedge from $s_{n-1}$ to $s_{k}$. We also stop expanding $G_{l}^{s}$ if we have expanded as many times as the relative loop bound of $l$. Note that $G_{l}^{s}$ contains at least two nodes, as the bus context at first loop iteration is always distinguished from the bus contexts in any other loop iteration.

It is worth mentioning that construction of $G_{l}^{s}$ is much less computationally intensive than a full unrolling of $l$. The bus context at the entry of $l$ quickly reaches a fixed-point and we can stop expanding $G_{l}^{s}$. In our experiments, we found that the number of nodes in $G_{l}^{s}$ never exceeds ten.

Generating separate ILP constraints: Using each flow graph $G_{l}^{s}$ for loop $l$, we generate ILP constraints to distinguish different bus contexts under which a basic block can be executed. In an abuse of notation, we shall use $w . i$ to denote that the basic block $i$ is reached with bus context $w . i$ when the immediately enclosing loop of $i$ is reached with bus context $w$ in any iteration. The following ILP constraints are generated to bound the value of $M_{l}^{w}$ :

$$
\begin{gathered}
\forall w \in \Omega_{l}: \sum_{x \in \Omega_{l}} M_{l}^{x \rightarrow w}=M_{l}^{w} \\
\forall w \in \Omega_{l}: M_{l}^{w}-1 \leq \sum_{x \in \Omega_{l}} M_{l}^{w \rightarrow x} \leq M_{l}^{w}
\end{gathered}
$$

$$
\sum_{w \in \Omega_{l}} M_{l}^{w}=N_{l . h}
$$

where $N_{l . h}$ denotes the number of times the header of loop $l$ is executed. Equations $10-11$ generate standard flow constraints from each graph $G_{l}^{s}$, constructed for loop $l$. Special constraints need to be added for the bus contexts with which the loop is entered at the first iteration and at the last iteration. If $w$ is a bus context with which loop $l$ is entered at the last iteration, $M_{l}^{w}$ is more than the execution count of outgoing flows (i.e. $M_{l}^{w \rightarrow x}$ ). Equation 11 takes this special case into consideration. On the other hand, Equation 12 bounds the aggregate execution count of all possible contexts $w \in \Omega_{l}$ with the total execution count of the loop header. Note that $N_{l . h}$ will further be involved in defining the CFG structural constraints, which relate the execution count of a basic block with the execution count of its incoming and outgoing edges [11]. Equations 10-12 do not ensure that whenever loop $l$ is invoked, the loop must be executed at least once with some bus context in $\Omega_{l}^{s}$. We add the following ILP constraints to ensure this:

$$
\forall w \in \Omega_{l}^{s}: M_{l}^{w} \geq N_{l . h}^{w . h}
$$

Here $N_{l . h}^{w . h}$ denotes the number of times the header of loop $l$ is executed with bus context $w$. The value of $N_{l . h}^{w . h}$ is further bounded by the CFG structural constraints.

The constraints generated by Equations 10-13 are sufficient to derive the WCET of a basic block in the presence of non-nested loops. In the presence of nested loops, however, we need additional ILP constraints to relate the bus contexts at different loop nests. Assume that the loop $l$ is enclosed by an outer loop $l^{\prime}$. For each $w^{\prime} \in \Omega_{l^{\prime}}$, we may get a different element $s_{0} \in \Omega_{l}^{s}$ and consequently, a different $G_{l}^{s}=\left(V_{l}^{s}, E_{l}^{s}\right)$ for loop $l$. Therefore, we have the following ILP constraints for each flow graph $G_{l}^{s}$ :

$$
\forall G_{l}^{s}=\left(V_{l}^{s}, E_{l}^{s}\right): \sum_{w \in V_{l}^{s}} M_{l}^{w} \leq \operatorname{bound}_{l} *\left(\sum_{w^{\prime} \in \operatorname{parent}\left(G_{l}^{s}\right)} M_{l^{\prime}}^{w^{\prime}}\right)
$$

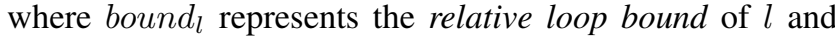
parent $\left(G_{l}^{s}\right)$ denotes the set of bus contexts in $\Omega_{l^{\prime}}$ for which the flow graph $G_{l}^{s}$ is constructed at loop $l$. The left-hand side of Equation 14 accumulates the execution count of all bus contexts in the flow graph $G_{l}^{s}$. The total execution count of all bus contexts in $V_{l}^{s}$ is bounded by bound $d_{l}$, for each construction of $G_{l}^{s}$ (as bound $d_{l}$ is the relative loop bound of l). Since $G_{l}^{s}$ is constructed $\sum_{w^{\prime} \in \operatorname{parent}\left(G_{l}^{s}\right)} M_{l^{\prime}}^{w^{\prime}}$ times, the total execution count of all bus contexts in $V_{l}^{s}$ is bounded by the right hand side of Equation 14.

Finally, we need to bound the execution count of any basic block $i$ (immediately enclosed by loop $l$ ), with different bus contexts. We generate the following two constraints to bound this value:

$$
\begin{gathered}
\sum_{w \in \Omega_{l}} N_{i}^{w . i}=N_{i} \\
\forall w \in \Omega_{l}: N_{i}^{w . i} \leq M_{l}^{w}
\end{gathered}
$$


where $N_{i}$ represents the total execution count of basic block $i$ and $N_{i}^{w . i}$ represents the execution count of basic block $i$ with bus context w.i. Equation 16 tells the fact that basic block $i$ can execute with bus context $w \cdot i$ at some iteration of $l$ only if $l$ is reached with bus context $w$ at the same iteration (by definition). $N_{i}$ will be further constrained through the structure of program's CFG, which we exclude in our discussion.

Computing bus contexts at loop exit: To derive the WCET of whole program, we need to estimate the bus context exiting a loop $l$ (say $O_{l}^{\text {exit }}$ ). A recently proposed work ([5]) has shown the computation of $O_{l}^{\text {exit }}$ without a full loop unrolling. In this paper, we use a similar technique as in [5] with one important difference: In [5], a single offset graph $G_{o f f}$ is maintained, which tracks the outgoing bus context from each loop iteration. Once $G_{o f f}$ got stabilized, a separate ILP formulation on $G_{\text {off }}$ derives the value of $O_{l}^{\text {exit }}$. In the presence of pipelined architectures, $O_{i}^{\text {out }}$ for any $i \in \pi_{l}^{\text {out }}$ could be responsible for propagating bus context outside of $l$ (refer to Figure 3 ). Therefore, a separate offset graph is maintained for each $i \in \pi_{l}^{\text {out }}$ (say $G_{o f f}^{i}$ ) and an ILP formulation for each $G_{o f f}^{i}$ can derive an estimation of the bus context exiting the loop (say $O_{i}^{e x i t}$ ). In [5], it has been proved that the computation of $O_{l}^{\text {exit }}$ is always an over-approximation (i.e. sound). Given that the value of each $O_{i}^{\text {out }}$ is sound, it is now straightforward to see that the computation of each $O_{i}^{\text {exit }}$ is also sound. For details of this analysis, readers are further referred to [5].

\section{EFFECT OF BRANCH PREDICTION ON CACHE}

Abstract-interpretation-based cache analysis produces a fixed point on abstract cache content at the entry (denoted as $A C S_{i}^{\text {in }}$ ) and at the exit (denoted as $A C S_{i}^{\text {out }}$ ) of each basic block $i$. If a basic block $i$ has multiple predecessors, output cache states of the predecessors are joined to produce the input cache state of basic block $i$. Consider an edge $j \rightarrow i$ in the program CFG. If $j \rightarrow i$ is an unconditional edge, computation of $A C S_{i}^{i n}$ does not require any change. However, if $j \rightarrow i$ is a conditional edge, the condition could be correctly or incorrectly predicted during the execution. For a correct prediction, the cache state $A C S_{i}^{i n}$ is still sound. On the other hand, for incorrect prediction, $A C S_{i}^{i n}$ must be updated with the memory blocks accessed at the mispredicted path. We assume that there could be at most one unresolved branch at a time. Therefore, the number of mispredicted instructions is bounded by the number of instructions till the next branch as well as the total size of instruction fetch queue and reorder buffer. To maintain a safe cache state at the entry of each basic block $i$, we join the two cache states arising due to the correct and incorrect predictions of conditional edge $j \rightarrow i$. We demonstrate the entire scenario through an example in Figure 4. In Figure 4 , we demonstrate the procedure for computing the abstract cache state at the entry of a basic block $i$. Basic block $i$ is

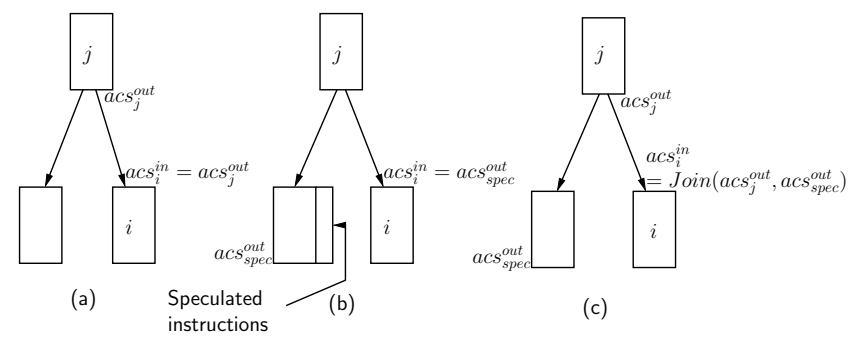

Figure 4. (a) Computation of $a c s_{i}^{i n}$ when the edge $j \rightarrow i$ is correctly predicted, (b) Computation of $a c s_{i}^{i n}$ when the edge $j \rightarrow i$ is mispredicted, (c) A safe approximation of $a c s_{i}^{i n}$ by considering both correct and incorrect prediction of edge $j \rightarrow i$.

conditionally reached from basic block $j$. To compute a safe cache content at the entry of basic block $i$, we combine two different possibilities - one when the respective branch is correctly predicted (Figure 4(a)) and the other when the respective branch is incorrectly predicted (Figure 4(b)). The combination is performed through abstract join operation, which on the other hand depends on the type of analysis (must or may) being computed. A stabilization on the abstract cache contents at the entry and exit of each basic block is achieved through conventional fixed point analysis.

\section{WCET COMPUTATION OF AN ENTIRE PROGRAM}

We compute the WCET of the entire program with $N$ basic blocks by using the following objective function:

Maximize $T=\sum_{i=1}^{N} \sum_{j \rightarrow i} \sum_{w \in \Omega_{i}} t_{j \rightarrow i}^{c, w} * E_{j \rightarrow i}^{c, w}+t_{j \rightarrow i}^{m, w} * E_{j \rightarrow i}^{m, w}$

$\Omega_{i}$ denotes the set of all bus contexts under which basic block $i$ can execute. Basic block $i$ can be executed with different bus contexts. However, the number of elements in $\Omega_{i}$ is always bounded by the number of bus contexts entering the loop immediately enclosing $i$ (refer to Section VI). $t_{j \rightarrow i}^{c, w}$ denotes the WCET of basic block $i$ when the basic block $i$ is reached from basic block $j$, the control flow edge $j \rightarrow$ $i$ is correctly predicted and $i$ is reached with bus context $w \in \Omega_{i}$. Similarly, $t_{j \rightarrow i}^{m, w}$ denotes the WCET of basic block $i$ under the same bus context but when the control flow edge $j \rightarrow i$ was mispredicted. Note that both $t_{j \rightarrow i}^{c, w}$ and $t_{j \rightarrow i}^{m, w}$ are computed during the iterative pipeline modeling (with the modifications proposed in Section V). $E_{j \rightarrow i}^{c, w}\left(E_{j \rightarrow i}^{m, w}\right)$ denotes the number of times basic block $i$ is reached from basic block $j$ with bus context $w$ and when the control flow edge $j \rightarrow i$ is correctly (incorrectly) predicted. Therefore, we have the following two constraints:

$$
E_{j \rightarrow i}^{c}=\sum_{w \in \Omega_{i}} E_{j \rightarrow i}^{c, w}, \quad E_{j \rightarrow i}^{m}=\sum_{w \in \Omega_{i}} E_{j \rightarrow i}^{m, w}
$$

Constraints on $E_{j \rightarrow i}^{c}$ and $E_{j \rightarrow i}^{m}$ are proposed by the ILP based formulation in [13]. On the other hand, $E_{j \rightarrow i}^{c, w}$ and $E_{j \rightarrow i}^{m, w}$ are bounded by the CFG structural constraints and the constraints proposed by Equations 10-16 in Section VI. 
Finally, the WCET of the program maximizes the objective function in Equation 17. Any ILP solver (e.g. CPLEX) can be used for the same purpose.

\section{SOUNDNESS OF ANALYSIS}

In this section, we shall provide the basic ideas for the proof of the soundness of our analysis framework. Due to space constraints, details of the proofs are included in the technical report [18].

The heart of soundness guarantee follows from the fact that we represent the timing of each pipeline stage as an interval. Recall that the active timing interval of each pipeline stage is captured by $I N T V_{i}=$ $\left[\right.$ earliest $\left[t_{i}^{\text {ready }}\right]$, latest $\left.\left[t_{i}^{\text {finish }}\right]\right]$. Therefore, as long as we can guarantee that $I N T V_{i}$ always over-approximates the actual timing interval of the corresponding pipeline stage in any concrete execution, we can also guarantee the soundness of our analysis. To ensure that the interval $I N T V_{i}$ is always an over-approximation, we have to consider all possible latencies suffered by any pipeline stage. The latency of a pipeline stage, on the other hand, may be influenced by the following factors:

Cache miss penalty: Only NC categorized memory references may have variable latencies. Our analysis represents this variable latency as an interval $[l o, h i]$ (Equation 1) where lo (hi) represents the latency of a cache hit (miss).

Functional unit latency: Some functional units may have variable latencies depending on the operands (e.g. multiplier unit). For such functional units, we consider the EX pipeline stage latency as an interval $[l o, h i]$ where $l o(h i)$ represents the minimum (maximum) possible latency of the corresponding functional unit.

Contention to access functional units: A pair of instructions may delay each other by contending for the same functional unit. Since only EX stage may suffer from contention, two different instructions may contend for the same functional unit only if the timing intervals of the respective EX stages overlap. For any pipeline stage $i$, an upper bound on contention (say $C O N T_{i}^{\max }$ ) is computed by accounting the cumulative effect of contentions created by all the overlapping pipeline stages (which access the same functional unit as $i$ ). We do not compute a lower bound on contention and conservatively assume a safe lower bound of 0 . Finally, we add $\left[0, C O N T_{i}^{\max }\right]$ with the timing interval of pipeline stage $i$. Clearly, $\left[0, C O N T_{i}^{\max }\right]$ covers all possible latencies suffered by pipeline stage $i$ due to contention.

Bus access delay: Bus access delay of a pipeline stage depends on the incoming bus contexts $\left(O_{i}^{i n}\right)$. Computation of $O_{i}^{i n}$ is always an over-approximation as evidenced by Equation 7 and Equation 9. Therefore, we can always compute the interval spanning from minimum to maximum bus delay using $O_{i}^{i n}$ (Equation 4 and Equation 5).

To conclude, we argue that the longest acyclic path search in the execution graph always results in a sound estimation of basic block WCET. Finally, the IPET approach searches for the longest feasible program path to ensure a sound estimation of whole program's WCET.

\section{EXPERIMENTAL EVALUATION}

We have chosen moderate to large size benchmarks from [9], which are generally used for timing analysis. Individual benchmarks are compiled into simplescalar PISA (Portable Instruction Set Architecture) [8] — a MIPS like instruction set architecture. The control flow graph (CFG) of each benchmark is extracted from its PISA compliant binary and is used as an input to our analysis framework.

To validate our analysis framework, the simplescalar toolset [8] was extended to support the simulation of shared cache and shared bus. The simulation infrastructure is used to compare the estimated WCET with the observed WCET. Observed WCET is measured by simulating the program for a few program inputs. Nevertheless, we would like to point out that the presence of a shared cache and a shared bus makes the realization of the worst case scenario extremely challenging. In the presence of a shared cache and a shared bus, the worst case scenario depends on the interleavings of threads, which are running on different cores. Consequently, the observed WCET result in our experiments may sometimes highly underapproximate the actual WCET.

For all of our experiments, we present the WCET overestimation ratio, which is measured as $\frac{\text { Estimated } W C E T}{\text { Observed } W C E T}$. Our analysis uses the default system configuration in Table I. Since the data cache modeling is not yet included in our current implementation, all data accesses are assumed to be Ll cache hits.

\begin{tabular}{|c||c|c|}
\hline Component & Default settings & Perfect settings \\
\hline Number of cores & 2 & NA \\
\hline pipeline & $\begin{array}{c}\text { 1-way, inorder } \\
\text { 4-entry IFQ, 8-entry ROB }\end{array}$ & NA \\
\hline $\begin{array}{c}\text { L1 instruction } \\
\text { cache }\end{array}$ & $\begin{array}{c}\text { 2-way associative, 1 KB } \\
\text { miss penalty = 6 cycles }\end{array}$ & $\begin{array}{c}\text { All accesses } \\
\text { are L1 hit }\end{array}$ \\
\hline $\begin{array}{c}\text { L2 instruction } \\
\text { cache }\end{array}$ & $\begin{array}{c}\text { 4-way associative, } 4 \mathrm{~KB} \\
\text { miss penalty = 30 cycles }\end{array}$ & NA \\
\hline Shared bus & slot length = 50 cycles & Zero bus delay \\
\hline $\begin{array}{c}\text { Branch predictor } \\
\text { 2 level predictor, L1 size }=1 \\
\text { L2 size=4, history size }=2\end{array}$ & $\begin{array}{c}\text { Branch prediction } \\
\text { is always correct }\end{array}$ \\
\hline \multicolumn{3}{|c|}{ Table I }
\end{tabular}

DEFAULT MICRO-ARCHITECTURAL SETTING FOR EXPERIMENTS

To check the dependency of WCET overestimation on the type of conflicting task (being run in parallel on a different core), we use two different tasks to generate the inter-core conflicts - 1) jfdctint, which is a single path program and 2) statemate, which has a huge number of paths. In our experiments (Figure 5(a)-(d)), we use jfdctint to generate inter-core conflicts to the first half of the tasks (i.e. matmult to nsichneu). On the other hand, we use statemate to generate inter-core conflicts to the second half of the tasks (i.e. edn to st). Due to the absence of any infeasible program path, inter-core conflicts generated by a single path program (e.g. jfdctint) can be more 


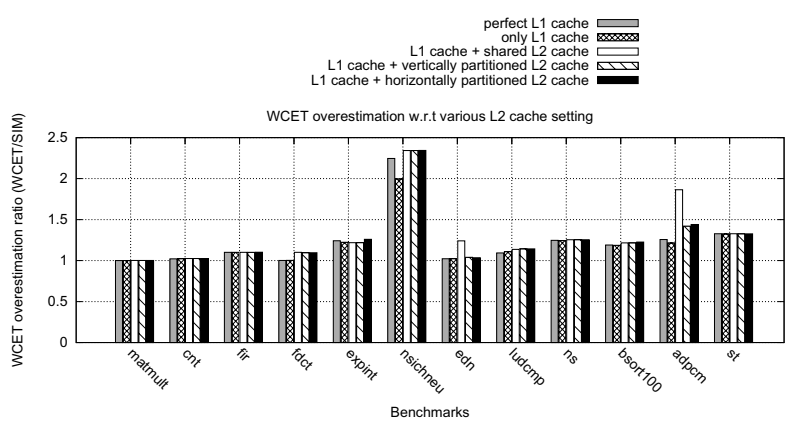

(a)

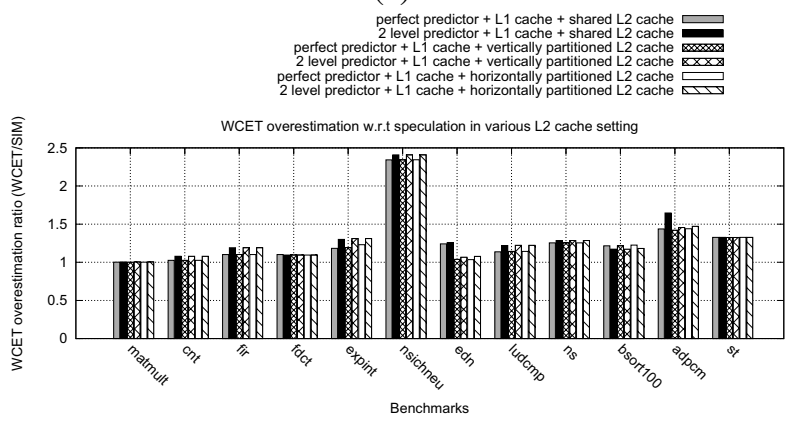

(c)

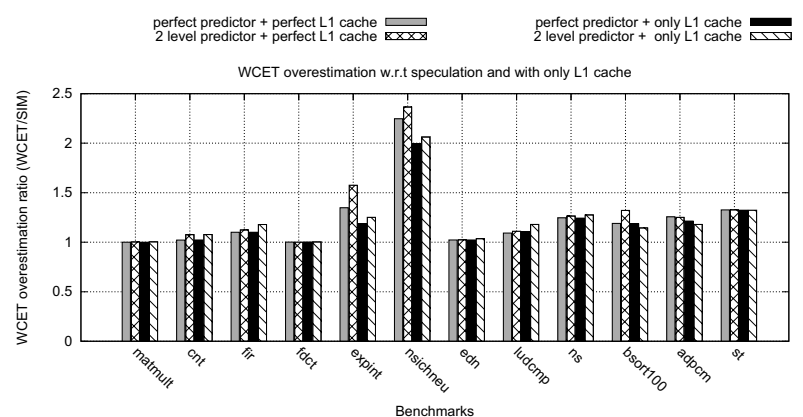

(b)

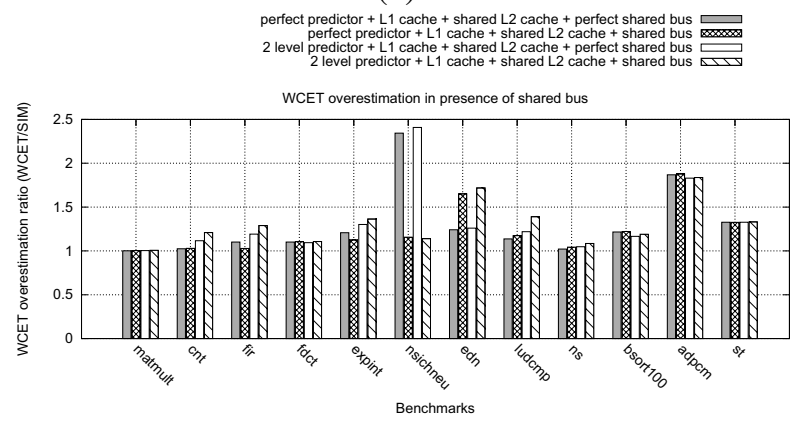

(d)

Figure 5. Effect of different micro-architectural parameters in WCET overestimation

accurately modeled compared to a multi-path program (e.g. statemate). Therefore, in the presence of a shared cache, we expect a better WCET overestimation ratio for the first half of the benchmarks (i.e. matmult to nsichneu) compared to the second half (i.e. edn to st).

To measure the WCET overestimation due to cache sharing, we compare the WCET result with two different design choices, where the level 2 cache is partitioned. For a two-core system, two different partitioning choices are explored: first, each partition has the same number of cache sets but has half number of ways compared to the original shared cache (called vertical partitioning). Secondly, each partition has half number of cache sets but has the same number of ways compared to the original shared cache (called horizontal partitioning). In our default configuration, therefore, each core is assigned a 2-way associative, $2 \mathrm{~KB}$ L2 cache in the vertical partitioning, whereas each core is assigned a 4-way associative, $2 \mathrm{~KB} \mathrm{L2}$ cache in the horizontal partitioning.

Finally, to pinpoint the source of WCET overestimation, we can selectively turn off the analysis of different microarchitectural components. We say that a micro-architectural component has perfect setting if the analysis of the same is turned off (refer to column "Perfect settings" in Table I).

Effect of caches: Figure 5(a) shows the WCET overestimation ratio with respect to different L1 and L2 cache settings in the presence of a perfect branch predictor and a perfect shared bus. Results show that we can reasonably bound the WCET overestimation ratio except for nsichneu. The main source of WCET overestimation in nsichneu comes from the path analysis and not due to the micro-architectural modeling. This is expected, as nsichneu contains more than two hundred branch instructions and many infeasible paths. These infeasible paths can be eliminated by providing additional user constraints into our framework and hence improving the result. We also observe that the partitioned L2 caches may lead to a better WCET overestimation compared to the shared L2 caches, with the vertical L2 cache partitioning almost always working as the best choice. The positive effect of the vertical cache partitioning is visible in edn and adpcm, where the overestimation in the presence of a shared cache rises. This is due to the difficulty in modeling the inter-core cache conflicts from statemate (a many-path program being run in parallel).

Effect of speculative execution: As we explained in Section VII, the presence of a branch predictor and speculative execution may introduce additional computation cycles for executing a mispredicted path. Moreover, speculative execution may introduce additional cache conflicts from a mispredicted path. The results in Figure 5(b) and Figure 5(c) show the effect of speculation in L1 and L2 cache, respectively. Mostly, we do not observe any sudden spikes in the WCET overestimation just due to speculation. adpcm shows some reasonable increase in WCET overestimation with L2 caches and in the presence of speculation (Figure $5(\mathrm{c})$ ). This increase in the overestimation ratio can be explained from the overestimation arising in the modeling of the effect of speculation in cache (refer to Section VII). 
Due to the abstract join operation to combine the cache states in correct and mispredicted path, we may introduce some spurious cache conflicts. Nevertheless, our approach for modeling the speculation effect in cache is scalable and produces tight WCET estimates for most of the benchmarks.

Effect of shared bus: Figure 5(d) shows the WCET overestimation in the presence of a shared cache and a shared bus. We observe that our shared bus analysis can reasonably control the overestimation due to the shared bus. Except for edn and nsichneu, the overestimation in the presence of a shared cache and a shared bus is mostly equal to the overestimation when shared bus analysis is turned off (i.e. a perfect shared bus). Experiments with nsichneu shows some interesting result. We observe that the WCET overestimation ratio decreases by a large factor when shared bus analysis is enabled. As we inspect the cause, we found that the execution time of nsichneu is dominated by shared bus delay, which is most accurately computed by our analysis for this benchmark. On the other hand, we observed in Figure 5(a) that the main source of WCET overestimation in nsichneu is path analysis, due to the presence of many infeasible paths. Consequently, when shared bus analysis is turned off, the overestimation arising from path analysis dominates and we obtain a high WCET overestimation ratio. Average WCET overestimation in the presence of both a shared cache and a shared bus is around $50 \%$.

WCET overestimation sensitivity: We have also conducted detailed experiments to check the WCET overestimation sensitivity with respect to L1 cache size, L2 cache size, bus slot length and different pipelines (inorder, out-of-order and superscalar). On average, our framework results around $40 \%$ overestimation and a maximum of $90 \%$ overestimation when very small $\mathrm{L} 1$ cache is used (512 bytes). For very small L1 cache size, our analysis results in L1 cache thrashing which eventually results in an overestimation of shared cache and shared bus traffic. Due to space constraints, details of these results are included in [18].

Analysis time: We have performed all the experiments on an 8 core, $2.83 \mathrm{GHz}$ Intel Xeon machine having $4 \mathrm{~GB}$ of RAM and running Fedora Core 4 operating systems. In most of the cases, our analysis finishes within a few seconds. ILP solver time dominates when branch prediction is enabled. When all the micro-architectural features are analyzed (pipeline, L1 and shared L2 cache, shared bus and branch prediction), our analysis takes maximum time (around 300 seconds) for the program nsichneu, with an average of 20-30 seconds over all other programs.

\section{Conclusion}

In this paper, we have proposed a sound WCET analysis framework by modeling different micro-architectural components and their interactions in a multi-core processor. Our analysis framework is also sound in the presence of timing anomalies. Our experiments suggest that we can obtain tight WCET estimates for the majority of benchmarks in a variety of micro-architectural configurations. Apart from design space exploration, we believe that our framework can be used to figure out the major sources of overestimation in multi-core WCET analysis. As a result, our framework can help in designing predictable hardware for real time applications and it can also help writing real time applications for the predictable execution in multi-cores.

\section{ACKNOWLEDGEMENT}

This work was partially funded by A*STAR Public Sector Funding Project Number 1121202007 - "Scalable Timing Analysis Methods for Embedded Software", and by the ArtistDesign Network of Excellence (the European Community's 7th Framework Program FP7/2007-2013 under grant agreement no 216008).

\section{REFERENCES}

[1] T. Lundqvist and P. Stenström. Timing anomalies in dynamically scheduled microprocessors. In Proceedings of the 20th IEEE Real-Time Systems Symposium, RTSS, 1999.

[2] J. Yan and W. Zhang. WCET analysis for multi-core processors with shared L2 instruction caches. In RTAS, 2008.

[3] Y. Li et. al. Timing analysis of concurrent programs running on shared cache multi-cores. In RTSS, 2009.

[4] S. Chattopadhyay, A. Roychoudhury, and T. Mitra. Modeling shared cache and bus in multi core platforms for timing analysis. In SCOPES, 2010.

[5] T. Kelter et. al. Bus aware multicore WCET analysis through TDMA offset bounds. In ECRTS, 2011.

[6] M. Lv et. al. Combining abstract interpretation with model checking for timing analysis of multicore software. In RTSS, 2010.

[7] X. Li et. al. Chronos: A timing analyzer for embedded software. Science of Computer Programming, 2007. http://www.comp.nus.edu.sg/ rpembed/chronos.

[8] T. Austin, E. Larson, and D. Ernst. Simplescalar: An infrastructure for computer system modeling. Computer, 35(2), 2002.

[9] WCET benchmarks. http://www.mrtc.mdh.se/projects/wcet/benchmarks.html

[10] Y-T. S. Li, S. Malik, and A. Wolfe. Performance estimation of embedded software with instruction cache modeling. ACM Trans. Des. Autom. Electron. Syst., 4(3), 1999.

[11] H. Theiling, C. Ferdinand, and R. Wilhelm. Fast and precise WCET prediction by separated cache and path analyses. Real-Time Systems, 18(2/3), 2000.

[12] X. Li, A. Roychoudhury, and T. Mitra. Modeling out-of-order processors for WCET analysis. Real-Time Systems, 34(3), 2006.

[13] X. Li, T. Mitra, and A. Roychoudhury. Modeling control speculation for timing analysis. Real-Time Systems, 29(1), 2005.

[14] D. Hardy, T. Piquet, and I. Puaut. Using bypass to tighten WCET estimates for multi-core processors with shared instruction caches. In RTSS, 2009.

[15] J. Rosen et. al. Bus access optimization for predictable implementation of realtime applications on multiprocessor systems-on-chip. In RTSS, 2007.

[16] M. Paolieri et. al. Hardware support for WCET analysis of hard real-time multicore systems. In ISCA, 2009.

[17] R. Pellizzoni et. al. A predictable execution model for COTS-based embedded systems. In RTAS, 2011.

[18] S. Chattopadhyay et. al. A unified WCET analysis framework for multicore platforms. http://www.comp.nus.edu.sg/ rpembed/chronos/publication/ chronos-multi-core.pdf. 